\title{
Die traumatische Aortenruptur
}

\author{
Thomas Schachner · Julia Oji-Zurmeyer (iD - Bartosz Rylski · Stephan Kapral • Olaf Stanger · Gerlig Widmann · \\ Florian Schönhoff · Martin Czerny · Stefan Jochberger
}

Zusammenfassung Verkehrsunfälle sind für den größten Teil der traumatischen Aortenrupturen verantwortlich, meist zusammen mit weiteren schweren Begleitverletzungen. Die prähospitale Sterblichkeit ist hoch. Bei Verdacht auf eine beteiligte Aortenverletzung, aufgrund eines hohen traumatic aortic injury scores, ist eine Computertomographie durchzuführen. Es erfolgt eine Triage der Verletzungen, und die Versorgungspriorität der Aorta richtet sich nach dem Schweregrad der Aorten- und Begleitverletzungen sowie dem Zustand des Patienten. Bis zur definitiven Versorgung der aortalen Läsion muss der Blutdruck konsequent gesenkt werden. Grad I und II können in Einzelfällen unter enger Kontrolle inital konservativ gemanagt werden. Grad III (gedeckte Ruptur) und Grad IV (freie Ruptur) benötigen eine raschestmögliche Versorgung (interventionell, chirurgisch).

\section{T. Schachner \\ Universitätsklinik für Herzchirurgie, Medizinische Universität Innsbruck, Anichstraße 35, 6020 Innsbruck, Österreich \\ thomas.schachner@i-med.ac.at}

Dr. J. Oji-Zurmeyer $(\bowtie) \cdot S$. Jochberger Universitätsklinik für Anästhesie und

Intensivmedizin, Medizinische Universität Innsbruck, Anichstraße 35, 6020 Innsbruck, Österreich

julia.oji-zurmeyer@tirol-kliniken.at

\section{S. Jochberger \\ stefan.jochberger@i-med.ac.at}

B. Rylski $\cdot$ M. Czerny

Klinik für Herz- und Gefäßchirurgie,

Universitäts-Herzzentrum Freiburg, Bad Krozingen,

79189 Bad Krozingen, Deutschland

\section{B. Rylski}

bartosz.rylski@uniklinik-freiburg.de

M. Czerny

martin.czerny@uniklinik-freiburg.de
In ausgesuchten Fällen kann auch eine verzögerte Versorgung günstig sein.

Als bevorzugte Versorgungsform hat sich die interventionelle Stentgrafttherapie etabliert.

Schlüsselwörter Traumatische Aortenruptur • Sterblichkeit · Versorgungskonzepte · Interventionelle Stentgraftherapie $\cdot$ Intensivmedizinische Therapie

\section{Traumatic aortic rupture - diagnosis and managment}

Summary Road traffic accidents are the main cause of traumatic aortic ruptures, mostly in combination with other severe injuries. The pre-hospital mortality rate is high. Suspected aortic trauma, following a high traumatic aortic injury score, is an indication for computer tomography. Injuries are triaged and the treatment priority of the aortic trauma is ascertained based on the severity of the aortic and concomitant injuries and

\footnotetext{
S. Kapral

Institut für Anästhesie und Intensivmedizin,

Unfallkrankenhaus Linz, Garnisonstraße 7, 4010 Linz,

Österreich

stephan.kapral@auva.at

O. Stanger

Klinik für Gefässchirurgie, Helios Kliniken München West, Steinerweg 5, 81241 München, Deutschland

olaf.stanger@helios-gesundheit.de

\section{G. Widmann}

Universitätsklinik für Radiologie, Medizinische Universität Innsbruck, Anichstraße 35, 6020 Innsbruck, Österreich gerlig.widmann@i-med.ac.at

F. Schönhoff

Universitätsspital Bern, Universitätsklinik für Herz- und Gefässchirurgie, Freiburgstraße 15, 3010 Bern, Schweiz florian.schoenhoff@insel.ch
} 
the condition of the patient. Until definitive treatment of the aortic lesion is completed, the blood pressure of the patient must be kept low. Grade I and II lesions can be managed under strict monitoring with initial conservative treatment in individual cases. Grade III (contained perforation) and grade IV (open rupture) lesions need surgical or interventional treatment as swiftly as possible. In selected cases, a delayed treatment can also be advantageous.

The endovascular stent graft therapy has established itself as the preferred form of treatment.

Keywords Traumatic aortic rupture - Mortality · Treatment concepts · Endovascular stent graft therapy · Intensive care

\section{Epidemiologie}

Unter allen tödlichen motorisierten Verkehrsunfällen in UK wurden in der Autopsie bei $20 \%$ der Opfer eine rupturierte Aorta gefunden [1]. Für die USA wurde geschätzt, dass von den jährlichen Unfallopfern im Straßenverkehr 7000-8000 an einer Aortenruptur versterben [2]. Auch bei einem schweren Zugunglück fanden sich unter den Unfallopfern viele mit Thoraxtrauma und einer Aortenverletzung [3]. Die Aortenruptur ist damit nach den Schädel-Hirn-Verletzungen die häufigste aller Todesursachen nach stumpfem Thoraxtrauma bei Verkehrsunfällen [4].

Insgesamt verursachen Verkehrsunfälle bis zu $90 \%$ der traumatischen Aortenrupturen [5]. Davon sind sämtliche Verkehrsteilnehmer und auch Fußgänger betroffen [6]. Während in selteneren Fällen die Aorta ascendens betroffen ist (meist schwerer Frontalzusammenstoß ohne Gurt), rupturiert sonst fast immer die proximale Aorta descensens unmittelbar nach Abgang der A. subclavia [7]. Der Aairbag kann davor keinen vollständigen Schutz bieten [8-12]. Unter den weiteren ursächlichen Unfällen ist der Sturz aus größerer Höhe (ab ca. 2. Stockwerk, Alpinunfall etc.) zu nennen.

Bis zu $90 \%$ der Unfallopfer mit Aortenruptur versterben noch am Unfallort [6, 8, 13]. Nur etwa 5-14\% der Betroffenen überleben zunächst am Unfallort und könnten potentiell ein Krankenhaus erreichen $[4,8,13]$. Die Gesamtletalität ist mit 94-98\% besonders hoch $[6,8,13,14]$. Da es sich zumeist nicht um isolierte Aortenverletzungen, sondern um Polytraumen handelt, sind die schnelle Diagnose und die optimale Behandlungsstrategie von größter Wichtigkeit.

\section{Pathomechanismus}

In den meisten Fällen der Verkehrstoten mit Aortenruptur (loco typico) handelt es sich um ein stumpfes Thoraxtrauma mit seitlicher Gewalteinwirkung [8, 13-16]. Noch präziser könnte die Kraft von ventral oder ventro-caudal auf den Thorax einwirken
[14, 16]. Diese plötzliche Dezeleration führt zu einer starken intrathorakalen Kompression mit kranialer Verschiebung des Mediastinums [8, 13, 17]. Die dabei an der Aorta auftretenden Dreh- und Scherkräfte können $\mathrm{zu}$ einer transversen Lazeration mit Ruptur führen [5]. Je abrupter und stärker das Dezelerationstrauma beim Unfall einwirkt, desto größer ist die Wahrscheinlichkeit für eine Ruptur. Mehr als 2/3 der Autoinsassen, $90 \%$ der Motorradfahrer und $70 \%$ der Fußgänger und Radfahrer mit Aortenruptur erlebten ein $\Delta_{\mathrm{v}}$ (Geschwindigkeitsdifferenz des Dezelerationstraumas, Aufprallgeschwindigkeit) $>50 \mathrm{~km} / \mathrm{h}$. Bei einem $\Delta_{\mathrm{V}}<50 \mathrm{~km} / \mathrm{h}$ erlitten $70 \%$ der Motorradfahrer keine Aortenruptur [14]. In diesem Geschwindigkeitsbereich könnte also eine kritische Wirkungskraft liegen.

Die häufigste Lokalisation der traumatischen Ruptur ist mit $\sim 90 \%$ der (immobile) Aortenisthmus (,loco typico") im proximalen Abschnitt der Aorta thoracica descendens distal der linken Arteria subclavia und mit einem charakteristischen queren Einriss [8, 13, 17]. Alle Hypothesen zur traumatischen Aortenruptur gehen von einem weitgehenden Zusammenwirken 1) der mechanischen Deformation, 2) einem intraluminalem Hochdruck, und 3) biomechanischer Eigenschaften der Aortenwand aus [18].

1. Die Deformation der Aorta (Scherkräfte, Zug, Spannung, Verdrehung, Verwindung, Biegung) kann dadurch verursacht werden, dass bei der plötzlichen Dezeleration die mehr flexiblen Anteile der proximalen Aorta und des Aortenbogens nach kranial ausweichen können, während die proximale Aorta descendens aber mit dem Isthmus an der Wirbelsäule fixiert ist und belastet wird [19].

Nach einer anderen Hypothese verhält sich die proximale Aorta unter lateraler Krafteinwirkung wie der lange Arm eines steifen Hebels mit der A. subclavia als Drehpunkt [20]. Dem kurzen Arm mit größerer Krafteinwirkung würde der Isthmus entsprechen.

Nach der „osseous pinch-Hypothese“ kommt es beim stumpfen Thoraxtrauma durch den Einschluss der Aorta zwischen Manubrium, erster Rippe, Klavicula und den Wirbelkörpern zu einer mechanischen Krafteinwirkung auf den Isthmus [21].

2. Eine intraluminale Druckerhöhung könnte ebenso an der Ruptur beteiligt sein. Am einfachsten ist die Vorstellung einer durch äußere Thoraxkompression erzeugten intravasalen Druckspitze. Unter experimentellen Bedingungen sind aber die für eine Ruptur notwendigen extremen Drücke alleine kaum erreichbar [18].

Die „water hammer-Hypothese“ besagt, dass ein stumpfes Thoraxtrauma zu einer Verengung der diaphragmalen Aortenöffnung führt und eine dadurch verursachte reflektorische Druckwelle am Bogen maximal einwirkt, gegebenfalls noch verstärkt durch die mediastinale Verschiebung [22]. Letztlich könnte eine 
plötzliche Blutdruckspitze auch durch eine stressbedingte Katecholaminfreisetzung beim Unfallhergang begünstigt sein [23].

3. Die Aorta hat anisotropische Eigenschaften mit einer vorwiegend zirkumferenten Steifigkeit [24]. Die biomechanische Zugfestigkeit in der transversen Richtung ist etwa doppelt so groß wie longitudinal und kann bei zusätzlicher Deformation zu querer Rissbildung führen [25]. Außerdem besitzen Media und Adventitia eine größere Elastizität als die endothelseitigen Wandanteile, weshalb diese steifere Struktur bei Druckspitzen von größerem Stress betroffen ist $[19,26]$.

\section{Diagnostik}

\section{Klinische Diagnostik und Klassifikationen}

Bei einer Ruptur im Isthmusbereich kann die Klinik ähnlich der einer Coarctatio sein, mit höherem Blutdruck am Arm im Vergleich zum Bein, einer Pulswellenverzögerung zwischen A. radialis und A. femoralis und einem scharfen rauen interskapulären Geräusch [13].

Meistens handelt es sich aber um polytraumatisierte Patienten, bei denen unter Umständen auch eine Aortenruptur vermutet werden muss [27]. Die häufigsten Begleitverletzungen sind Frakturen ( 70\%), Thoraxverletzungen ( $50 \%)$, abdominale Verletzungen $(\sim 40 \%)$ und Kopfverletzungen ( 37\%) [13]. Um die Wahrscheinlichkeit für das Vorliegen einer Aortenruptur besser einschätzen zu können, wurde bei Traumapatienten mit einem injury severity score (ISS) > 15 der ,traumatic aortic injury score“ (TRAINS) entwickelt und verschiedene prädiktive Kriterien für das Vorliegen einer Aortenruptur identifiziert:

- Erweitertes Mediastinum ${ }^{1}: 4$ Punkte

- Hypotension $\left(<90 \mathrm{~mm} \mathrm{Hg}^{2}{ }^{2}: 2\right.$ Punkte

- Fraktur proximaler langer Knochen: 2 Punkte

- Lungenkontusion: 1 Punkt

- Fraktur der linken Skapula: 1 Punkt

- Hämothorax: 1 Punkt

- Beckenfraktur - Deformität: 1 Punkt

Ab einem Score von $\geq 4$ Punkten liegt ein erhöhtes Risiko für eine Aortenruptur vor und erfordert eine sofortige CT-Untersuchung [28].

Zusätzliche Klassifikationen beziehen sich auf den Schweregrad der Aortenverletzung und können bei der Planung des weiteren Vorgehens hilfreich sein [29]:

- Typ I: Intimaeinriss

- Typ II: Intramurales Hämatom (IMH)

\footnotetext{
1 Mediastinum $>8 \mathrm{~cm}$ oder $>25 \%$ der Thoraxbreite auf Höhe des Aortenknopfes im Thoraxröntgen.

2 Oder Notwendigkeit einer Flüssigkeits- oder Katecholamingabe zum Erhalt eines Blutdrucks $>90 \mathrm{~mm} \mathrm{Hg}$
}

- Typ III: Pseudoaneurysma

- Typ IV: Ruptur

Höhere Schweregrade der Aortenruptur sind mit einem ausgeprägteren Gesamtverletzungsbild assoziiert. Bei einem Intimariss $<1 \mathrm{~cm}$ Länge war der mittlere ISS-Score 36,5 , bei einem größeren Intimariss $(>1 \mathrm{~cm}$ ) von 36,6 , bei einem Pseudoaneurysma bzw. einer gedeckter Ruptur 40,4 und bei einer freien Ruptur 55,6. [30].

Weil der Erkrankungsverlauf eine deutliche Abhängigkeit vom radiologischen Schweregrad der Aortenruptur zeigt, wurde eine weitere Klassifikation von der Society of Vascular Surgery vorgeschlagen [30]:

- Grad 1: Intimariss oder assoziierter Thrombus $<1 \mathrm{~cm}$

- Grad 2: Intimariss oder assoziierter Thrombus $>1 \mathrm{~cm}$

- Grad 3: Konturverlust der Aorta, gedeckte Ruptur

- Grad 4: Konturverlust der Aorta, freie Ruptur (i.e. Kontrastmittelaustritt)

Nur in seltenen Fällen wird die Ruptur überlebt. Die Läsion kann dann sogar lange unbemerkt bleiben, und es kann sich in bis zu Jahren später ein falsches Aneurysma bilden.

Bei Grad I und II (ohne äußere Konturveränderung) treten kaum frühe Todesfälle auf, und es wurde in einigen Zentren auch primär konservativ behandelt [30, 31]. Bei etwa der Hälfte der Patienten kann sogar eine Befundbesserung bzw. eine komplette Auflösung des radiologischen Befundes erwartet werden. Bei $40 \%$ der Patienten stabilisiert sich der Befund, und nur bei ca. 5\% kam es zu einer Befundprogression [31].

Für Patienten mit Grad III (gedeckte Ruptur) und IV (freie Ruptur) besteht aufgrund der hohen Komplikationsrate und schlechten Prognose die Indikation zur raschestmöglichen chirurgischen oder endovaskulären Therapie [30]. Zusätzliche Indikationen sind ein periaortales Hämatom $>15 \mathrm{~mm}$ und eine Progression des Schweregrades [30].

\section{Bildgebende Diagnostik}

Die lange gebräuchliche Aortographie ist inzwischen vollständig von der Ganzkörper-Computer-Tomographie (CT) abgelöst worden. Bei einem polytraumatisierten Patienten wird nach dem bereits genannten TRAINS-Score eine Sensitivität von $93 \%$ und eine Spezifität von $86 \%$ zur Diagnose einer Aortenruptur erreicht [28]. Das Ganzkörper-CT erlaubt die schnelle Diagnose und Schweregradeinteilung der Aortenruptur [32] sowie die Beurteilung sämtlicher anderer Organe für eine Triagierung des weiteren Vorgehens. Weitere häufige relevante Befunde sind mediastinales Hämatom, Hämatothorax, Pseudoaneurysma, Intimariss und Thrombusformation. Schließlich kann sofort eine 3D-Rekonstruktion durchgeführt werden, um eine endovaskuläre Behandlung mit TEVAR planen $\mathrm{zu}$ können. 


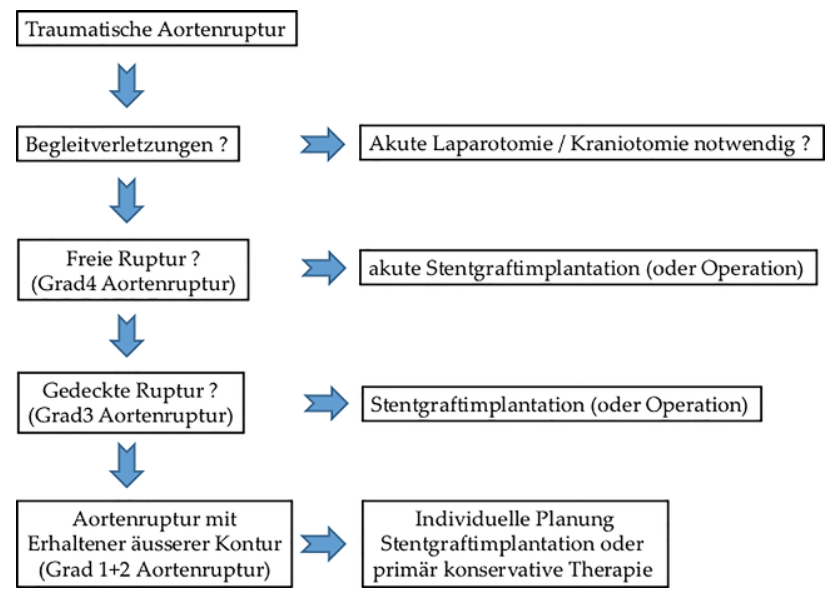

Abb. 1 Behandlungspfad bei traumatischer Aortenruptur

Ein alleiniges Röntgenbild vom Thorax ist für eine Diagnose völlig unzureichend und kann bestenfalls, jedoch mit häufigen falsch-positiven Befundungen, einen indirekten Hinweis auf eine Mediastinumverbreiterung liefern $[8,33,34]$.

Als wichtige Ergänzung zum CT ist die transösophageale Echokardiographie (TEE) von großer Bedeutung. Für den Nachweis einer Aortenverletzung wurde eine Sensitivität von 91-100 \% und Spezifität bis 98-100\% angegeben, aber die Notfallsituation beim polytraumatisierten Patienten lässt die Untersuchung nicht immer zu [35, 36]. Abdominelle Begleitverletzungen können nicht gleichzeitig untersucht werden. Dafür wichtig zum Monitoring bei drohender Perikardtamponade und begleitenden Intimaflaps und Dissektionen [37].

Realistischerweise hängt die Untersuchungsmöglichkeit aber oft vom Zustand und der Stabilität des Patienten ab, sowie von den Dringlichkeit, Begleitverletzungen zu erfassen.

\section{Behandlung}

Der Spontanverlauf der traumatischen Aortenruptur ist einerseits vom Ausmaß der Begleitverletzungen abhängig, und andererseits vom Typ und Schweregrad der Aortenruptur. Diese bestimmen auch grundsätzlich den Interventionszeitpunkt. Unter Umständen kann die Intervention auch bis zu $24 \mathrm{~h}$ aufgeschoben werden, um den Patienten zunächst in einen hämodynamisch stabilen Zustand für die Aortenintervention zu bringen.

Bei Patienten mit relativ geringem Verletzungsausmaß (Grad I und II) kann, unter engmaschigen CTKontrollen, in individueller Abwägung initial konservativ vorgegangen werden. Die Entscheidung zur zweitrangigen oder aufgeschobenen Versorgung der Aortenruptur ist hier beim polytraumatisierten $\mathrm{Pa}$ tienten unter Einbezug der Begleitverletzungen individuell $\mathrm{zu}$ treffen. Andere lebensbedrohliche und akut blutende Verletzungen sind unter Umständen vorrangig zu behandeln. In Studien blieben darunter die meisten dieser Läsionen entweder stabil und oder waren regredient. Nur ein kleiner Teil $(\sim 5 \%)$ zeigte in den Kontrollen eine Progredienz [31, 38, 39]. Wenn eine höhergradige Aortenverletzung (>Grad 2; Zeichen der gedeckten oder freien Ruptur oder periaortales Hämatom) vorliegt, eine Konturveränderung der Aorta oder Komplikationen wie bspw. ein Hämatothorax auftreten, müssen die Patienten als Notfall sofort behandelt werden. Die Behandlung erster Wahl ist die Stentgraft-Implantation zur endovaskulären Abdeckung der rupturierten Aortenstelle.

Etwa 2/3 der Patienten mit traumatischer Aortenruptur haben auch noch weitere Begleitverletzungen (thorakal, abdominell oder kranial). Diese beeinflussen wesentlich die Überlebensprognose und erfordern einen individuell angepassten Behandlungsplan. Beispielsweise erscheint es in der Behandlungspriorität günstiger zu sein, die Aortenruptur bei gleichzeitigem Schädel-Hirn-Trauma erst verzögert ( $>24 \mathrm{~h}$ ) zu behandeln. Der Grund dafür ist, dass sich ansonsten das Hirntrauma verschlechtern kann [40].

Traumatische Rupturen der Aorta ascendens und des Aortenbogens kommen zwar seltener vor, sind aber häufig von zusätzlichen Verletzungen des Herzens begleitet (Herzkontusion, Aortenklappeninsuffizienz, Hämoperikard) und haben eine Hospitalletalität von $>50 \%$ [41]. Sie stellen prinzipiell eine herzchirurgische Notfallindikation dar [42].

Der Behandlungspfad der traumatischen Aortenruptur in Abhängigkeit vom Schweregrad der Ruptur ist in Abb. 1 dargestellt.

Der „optimale“ Zeitpunkt für die Behandlung der Aortenruptur ist immer noch nicht ganz geklärt und ist sehr individuell zu beurteilen.

Trotz hämodynamischer Stabilität wurde die Notfall-Operation meist durch die innerhalb von $24 \mathrm{~h}$ erwartete Ruptur gerechtfertigt. Inzwischen wird die Behandlungspriorität bei Begleitverletzungen differenzierter gesehen und die etwas aufgeschobene Versorgung der Aorta kann in selektierten Fällen im Ergebnis (Letalität- und Paraplegieraten) überlegen sein [43, 44].

\section{Konservative Behandlungsprinzipien}

Beim polytraumatisierten Patienten mit Aortenruptur ist ein multidisziplinäres Management mit der richtigen Prioritätensetzung entscheidend. Die initial konservative Behandlung ist für unkomplizierte akute Aortensyndrome der Aorta descendens sicher möglich und etabliert [42]. Als Sonderform ist das bei den milden Formen der traumatischen Aortenruptur ebenfalls möglich. Dabei nimmt eine konsequente Senkung des mittleren arteriellen Blutdrucks (mean arterial pressure; MAP) $<80 \mathrm{~mm} \mathrm{Hg}$ sein, v.a. mit $\beta$-Blockern, einen besonders wichtigen Stellenwert ein. Dadurch gelangen mehr Patienten als früher zu einer (unter Umständen verzögerten) Intervention 
[45]. Um das Risiko für eine Koagulopathie mit Blutung, Hypertension und Ruptur zu minimieren, sollte mit der Flüssigkeitszufuhr äußerst zurückhaltend verfahren werden $[2,29,46]$.

Bei einem primär konservativen Vorgehen (Grad I und II) ist eine Kontroll-CT-Untersuchung innerhalb $48 \mathrm{~h}$, und danach nochmalig nach $48 \mathrm{~h}$ indiziert, da in den ersten $72 \mathrm{~h}$ eine erhöhte Rupturgefahr besteht. Eine Progression des Schweregrades stellt, insbesonders bei Verlust der äußeren Kontur der Aorta, eine Indikation für eine interventionelle (Stentgraft) oder operative Behandlung dar.

\section{Offene Chirurgie}

Der erste Eingriff mit Interposition eines Homografts wurde 1951 durchgeführt [47]. Inzwischen wird das rupturierte Aortensegment mit einer Rohrprothese ersetzt. Früher wurde stets die sofortige Operation angestrebt, inzwischen hat sich aber ein individuelles Vorgehen etabliert. Sämtliche Verletzungen werden in der Behandlungsabfolge triagiert und die Versorgung der Aortenverletzung ggf. verzögert, aber unter möglichst stabilen Bedingungen noch innerhalb von $24 \mathrm{~h}$ durchgeführt [43, 44, 48-51]. Besonders bei jungen Patienten wäre eine definitive Therapie wünschenswert, welche immer noch im prothetischen Ersatz des betroffenen Aortensegments besteht.

Der operative Zugang zum Aortenisthmus führt über eine Thorakotomie im linken 4. Intercostalraum mit rechtsseitiger Lungenventilation. Die Aorta wird proximal der A. subclavia und distal der Ruptur geklemmt und das Segment mit dem Interponat versorgt.

Trotz der zügigen Technik mit „clamp-and-sew“ wurde die Mortalität bei diesem Eingriff mit 5,8-23\%, und die Paraplegierate mit 5-19\% angegeben [51-55]. Die distale Aortenperfusion mit extrakorporaler Zirkulation hat die perioperative Paraplegierate auf $3 \%$ senken können [52, 56]. In zwei Metaanalysen mit insgesamt 2338 Patienten hatte die offene Operation mit EKZ die höchste Mortalität (16,7-18,2\%), gefolgt von einem Shunt (11,4-12,3\%) [51, 52], jeweils ohne Unterschied bei der postoperativen Paralyserate [51, 57]. Als wichtigstes Kriterium für eine erfolgreiche Operation wurde eine möglichst kurze Klemmzeit von $<45$ min ermittelt [51, 58-61].

Die sehr guten Ergebnisse mit Links- und partiellem Rechtsherz-Bypass von 3,5-4\% Mortalität und ohne Fall von Paraplegie dürfte nur von sehr wenigen besonders spezialisierten und erfahrenen Zentren erreicht werden [62-64].

Traumatische Rupturen der Aorta ascendens und des Aortenbogens kommen zwar seltener vor, sind aber häufig von zusätzlichen Verletzungen des Herzens begleitet (Herzkontusion, Aortenklappeninsuffizienz, Hämoperikard) und haben eine Hospitalletalität von $>50 \%$ [41]. Sie stellen prinzipiell eine herzchirurgische Notfallindikation dar [42].

\section{TEVAR}

In den 1990er Jahren wurde eine Technik zur endovaskulären Ausschaltung von Aneurysmen entwickelt. Die erste endovaskuläre Stent-graft Versorgung der Aorta descendens wurde 1987 durchgeführt [65]. Seither wurde die Stentversorgung zunächst lediglich als Überbrückungsmaßnahme bis zur definitiven chirurgischen Versorgung betrachtet; tatsächlich aber kam es zwischenzeitlich zu einem Paradigmenwechsel, und die Intervention mit thoracic endovascular aortic repair (TEVAR) ist zur bevorzugten Behandlungsform der traumatischen Aortenruptur geworden [66, 67]. Im Vergleich mit der offenen Operation haben mehrere Metaanalysen Vorteile für die endovaskuläre Versorgung gezeigt; etwa eine deutlich niedrigere prozedurale und 30-Tage Letalitätsrate mit 9-19\% vs. $19-36 \%$, Paraplegie $3 \%$ vs. 5,5.-9\%, Nierenversagen $3 \%$ vs. $8 \%$, Graft-Infektion $3 \%$ vs. $11 \%$, systemische Infektion $5 \%$ vs. $13 \%$ [43, 44, 68, 69]. Dabei konnte für zerebralen Insult kein Unterschied erkannt werden (jeweils 3-10,2\%).

Diese Daten stammen jedoch fast ausschließlich von nicht-vergleichenden Fallserien, retrospektiven und nicht-randomisierten Studien. Der case mix (Verteilung der Schweregrade), nicht standardisierte Operationsverfahren und Patientenselektion sowie Publikationsbias erklären teilweise sehr unterschiedliche Ergebnisse und erschweren einen detaillierten Vergleich beider Verfahren [43, 64]. Abb. 2 zeigt den typischen Fall einer Grad 3 Ruptur mit endovaskulärer Versorgung.

Trotz den Einschränkungen bei den berichteten Daten ist TEVAR aufgrund der deutlich geringeren prozeduralen Letalität meist zum bevorzugten Verfahren geworden [44]. Dennoch müssen bei der Behandlung mittels Stentgraftimplantation zahlreiche Komplikationsmöglichkeiten berücksichtigt werden, und ca. $16 \%$ der Patienten müssen sich meist innerhalb des ersten Jahres einer Re-Intervention unterziehen [69, 70]. Entsprechend den Guidelines der Cardiovascular and Interventional Radiological Society of Europe (CIRSE) wird für die endovaskuläre Versorgung mittels Stentgraft eine proximale Landungszone von $20 \mathrm{~mm}$ verlangt, um eine gute Apposition $\mathrm{zu}$ erreichen und sowohl Endoleaks als auch eine Migration des Stentgrafts zu verhindern [71]. Eine Distanz von weniger als $20 \mathrm{~mm}$ zwischen dem Abgang der linken A. subclavia und der Rupturstelle bedarf einer Überstentung der A. subclavia mit oder ohne chirurgische Revaskularisation mit einem Carotis-SubclaviaBypass oder Transposition [72].

Die Überstentung ohne Revaskularisation wird in der Regel gut toleriert, kann aber in Einzelfällen zu Durchblutungsstörungen des linken Armes oder neurologischen Komplikation führen [73]. Die Darstellung der linken A. vertebralis kann hierfür hilfreich sein. Bei einem Durchmesser von $<3 \mathrm{~mm}$ muss mit einer schweren Claudicatio gerechnet werden [32, 74]. Es 
Abb. 2 a, b 51 Jahre alter Mann mit traumatischer Aortenruptur loco typico bei Z. n. Motorradunfall mit begleitender Leberruptur und Beckenfraktur. Gedeckte Ruptur mit Pseudoaneurysma und periaortalem Hämatom. c-f Typische interdisziplinäre endovaskuläre Versorgung mittels Stentgraft. $\mathbf{f}, \mathbf{g}$ Kontroll-CT nach 6 Monaten ohne Hinweis für Endoleaks
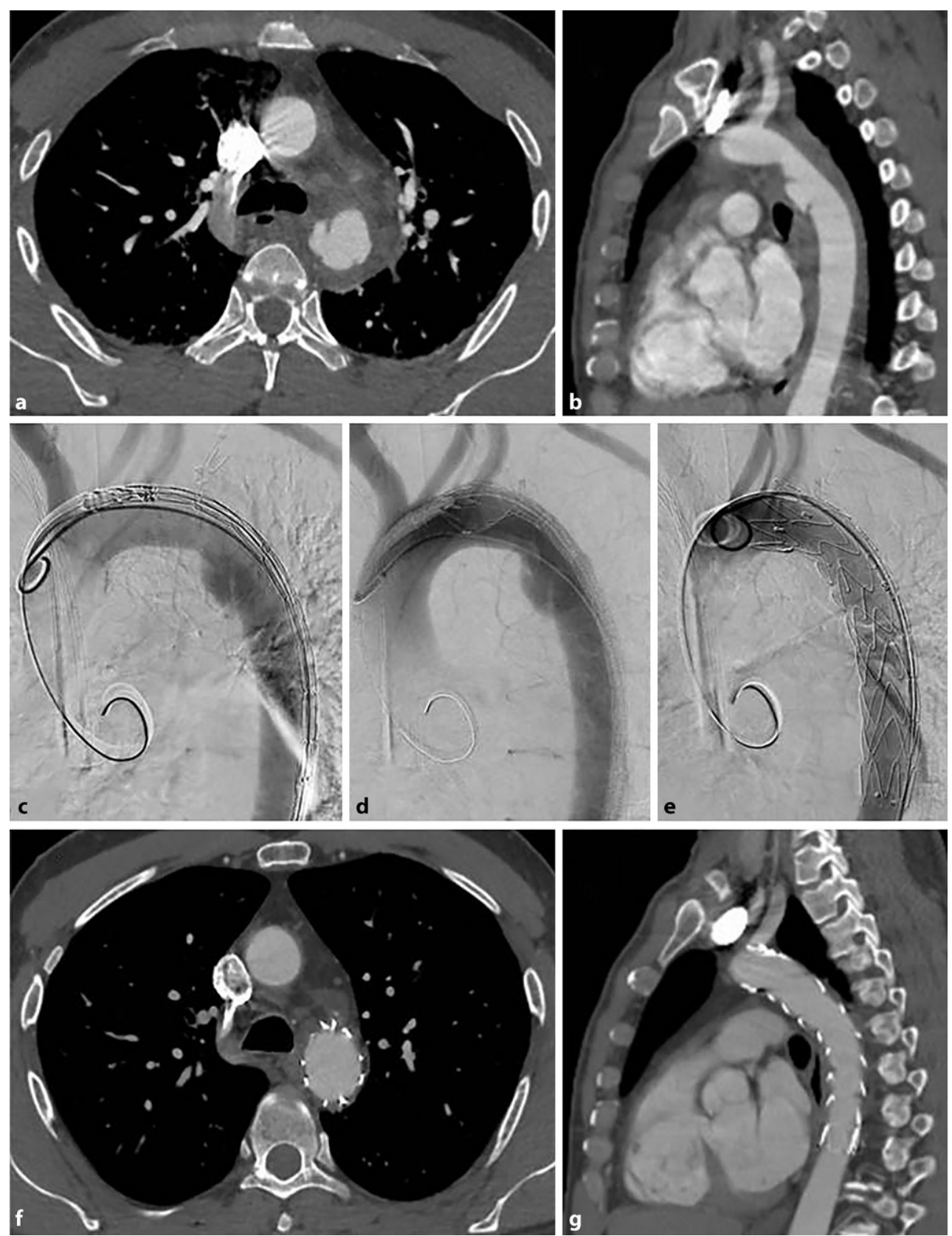

empfiehlt sich auch in einer CT-Angiographie die Circulus Willisi Durchgängigkeit darzustellen und, davon abhängig, eine Revaskularisierung der A. subclavia durchzuführen.

Weitere Komplikationen sind Endoleak (5-7\%) und Stentkollaps, seltener auch intraoperative Rupturen, Stentpenetration, Verletzungen der A. liaca, und Fistelbildungen zum Ösophagus [43, 50, 68, 69, 75-79].

Obwohl Guidelines TEVAR für die Behandlung der akuten und chronischen Aortenruptur grundsätzlich empfehlen, wird auf die fehlenden Daten zu Langzeitergebnissen hingewiesen [80]. Jüngere Patienten betrachten sich mitunter nicht als definitiv versorgt und benötigen eine lebenslange Beobachtung mit wiederholten CT-Untersuchungen und entsprechen- der Strahlen- und Kontrastmittelbelastung [81]. Es ist auch unklar, wie sich die „normale“ Größenzunahme der Aorta über einen längeren Zeitraum auswirken wird. Innerhalb der ersten 10 Jahren ist im Bereich der proximalen Landezone mit einer durchschnittlichen Aortaerweiterung von $3,3 \pm 1,5 \mathrm{~mm}$ zu rechnen [82].

Weitere Entwicklungen endovaskulärer Techniken mit gebranchten und fenestrierten Stentgrafts bzw. Snorkel, Periscope- oder Chimney-Techniken bleiben vorerst noch abzuwarten [83]. 
Tab. 1 Zielparameter der postoperativen Kreislauftherapie der S3-Leitlinie zur intensivmedizinischen Versorgung herzchirurgischer Patienten

$\mathrm{S}_{\mathrm{cv}} \mathrm{O}_{2}>70 \%$ oder $\mathrm{S}_{\mathrm{v}} \mathrm{O}_{2}>65 \%$
$\mathrm{MAP}>65 \mathrm{~mm} \mathrm{Hg}$
ZVD $8-12 \mathrm{~mm} \mathrm{Hg}$ (abhängig von der Beatmung)
Diurese $>0,5 \mathrm{ml} / \mathrm{kgKG} / \mathrm{h}$
Laktat $<3 \mathrm{mmol} / \mathrm{I}$
Cardiac Index $>2,0 \mathrm{l} / \mathrm{min} / \mathrm{m}^{2}$
LV-EDAl $6-9 \mathrm{~cm}^{2} / \mathrm{m}^{2}$
ITBVI $850-1000 \mathrm{ml} / \mathrm{m}^{2}$
GEDVI $640-800 \mathrm{ml} / \mathrm{m}^{2}$
PAOP $12-15 \mathrm{~mm} \mathrm{Hg}$

$\mathrm{ScvO}_{2}$ Central venous oxygen saturation (Zentralvenöse Sauerstoffsättigung), $\mathrm{S}_{V} \mathrm{O}_{2}$ Mixed venous oxygen saturation (Gemischtvenöse Sauerstoffsättigung), MAP Mean arterial pressure (Mittlerer arterieller Druck), ZVD Zentralvenöser Druck, LV-EDAI Left Ventricular End-Diastolic Area Index, ITBVI Intrathorakaler Blutvolumenindex, GEDVI Globales enddiastolisches Volumen, PAOP Pulmonary artery occlusion pressure

\section{Intensivmedizinisches Management}

Das intensivmedizinische Management von Patienten mit Aortenruptur ist abhängig von der vorausgegangenen chirurgischen Behandlung. Eine konservative Behandlung, ein endovaskuläres Verfahren mit Aortenstenting (TEVAR) oder eine offene Operation an der Herz-Lungen-Maschine initiieren teilweise erheblich unterschiedliche postoperative Verläufe. Es können lange oder kürzere Aufenthalte mit mehr oder weniger medizinischem Aufwand auf einer Intensivstation notwendig sein. Erschwerend können auch Begleitverletzungen bei der traumatischen Ätiologie der Aortenruptur eine Rolle spielen.

Grundelemente der intensivmedizinischen Therapie sind das hämodynamische Monitoring, eine adäquate Volumensubstitution, sowie der gezielte Einsatz von vasoaktiven und kardial wirksamen Medikamenten. Beim Zusammenspiel dieser wesentlichen Elemente sind eine ausreichende Gewebsperfusion sowie die Normalisierung des oxidativen Metabolismus das Ziel [1]. Dabei bedingen das intravasale Volumen und die kardiale Pumpfunktion ein ausreichendes HerzZeit-Volumen und in weiterer Folge ein ausreichendes Sauerstoff-Angebot im Gewebe.

Die Zielparameter der postoperativen Kreislauftherapie der S3-Leitlinie zur intensivmedizinischen Versorgung herzchirurgischer Patienten entsprechend den Kriterien des Oxford Centre for Evidenced-Based Medicine sind in Tab. 1 dargestellt [1].

Das Erhalten genannter Parameter im Zielbereich bedarf zumeist multipler Monitoringverfahren. Das postoperative Basismonitoring für Intensivpatienten umfasst die Pulsoxymetrie, die Elektrokardiographie, die invasive Blutdruckmessung, die Messung des zentralen Venendrucks, eine sorgfältige Bilanzierung (Drainagen, Ein- und Ausfuhr), arterielle und zentralvenöse Blutgasanalysen und eine Temperaturmes- sung. Der „geschulte klinische Blick“ der behandelnden Ärzte ist zugleich von großer Bedeutung.

Ergänzend können nach kritischer klinischer Abwägung und apparativer Verfügbarkeit erweiterte hämodynamische Monitoringverfahren eingesetzt werden. Diese dienen vor allem der Einschätzung des Herzzeitvolumens und der funktionellen Hämodynamik. Dazu zählen unter anderem die Echokardiographie (TTE/TEE), Thermodilutionsverfahren, Pulskontouranalysen, Stroke Volume Variation (SVV), Systolic-Pressure-Variation (SPV), Pulse Pressure Variation (PPV) und klinische Tests wie der passive Beinhebeversuch und Rekapillarisationstest.

Eine Expertise der behandelnden Ärzte in der Echokardiographie hat sich bewährt. Sie ist häufig entscheidend, um Volumenreagibilität und hämodynamischen Unterstützungsbedarf in Anbetracht vielerlei Messparameter abzuschätzen.

Bei der Vielfalt an zur Verfügung stehenden Verfahren ist es schwierig, allgemein gültige Empfehlungen abzugeben. Ausgesprochen sinnvoll und gegebenenfalls sogar prognostisch relevant ist ein individuell ausgerichtetes Monitoring für die patientenbezogene Therapiesteuerung. Diese kann zur sinnvollen Reduktion einer Katecholamintherapie führen, da Katecholamine in Abhängigkeit von Dauer und Dosierung zu adversen kardialen Ereignissen führen [84]. Dabei sollte ein Pulmonaliskatheter beispielsweise nur ausgewählt zur Anwendung kommen, da kein zusätzlicher Nutzen hinsichtlich einer reduzierten Letalität gezeigt werden konnte.

Der mittlere arterielle Blutdruck, die zentralvenöse Sättigung, der zentrale Venendruck, die Diurese und die Laktatkonzentration sind leicht erfassbare Parameter des Basismonitorings und damit primär messbare Ziele der Kreislauftherapie. Sie bilden die ersten Grundpfeiler zur Vermeidung einer Fehlverteilung des Blutflusses vor allem auf Mikrostrombahnebene. Häufigste Ursache dessen ist eine inadäquate kapilläre Perfusion und damit ein unzureichendes Sauerstoffangebot auf zellulärer Ebene.

Rezente Studien zeigen, dass mikrozirkulatorische Veränderungen im Kreislaufschock teilweise sogar unabhängig von systemischen Variablen sind [85]. Ein Monitoring der peripheren Perfusion kann auf den Effekt neurohumoraler Kompensationsmechanismen infolge von Schock hindeuten und entscheidende und frühe prognostische Hinweise geben [86].

Dennoch kann die unkritische Therapie mit vasoaktiven Substanzen, um den mittleren arteriellen Blutdruck über einen arbiträren Grenzwert anzuheben, zu unvorhersehbaren, negativen Effekten im Bereich der Mikrozirkulation und bei der Organfunktion führen [87]. Die postoperative Laktatkonzentration spielt hier eine entscheidende Rolle. Aber auch ein geschulter „klinischer Blick“, Zeichen wie verlängerte Rekapillarisationszeit $(>4,5 \mathrm{~s})$, Hautmarmorierung und kalte Extremitäten, kommen hier ins Spiel. Die Assoziation einer postoperativen Laktatazidose, also 
ein Zustand mit erhöhten Laktatwerten und einer metabolischen Azidose, hat sich als ein prädiktiver Faktor hinsichtlich einer erhöhten Letalität erwiesen $[88,89]$.

Das Gerinnungsmanagement und der Transfusionsbedarf richten sich nach der Höhe des Blutverlustes. Je nach Patientenprofil empfiehlt sich, ein Ziel-Hb von 7-9g/dl durch allogene Bluttransfusion anzustreben [90, 91]. Für das adäquate Funktionieren der Gerinnungskaskade ist das Aufrechterhalten von Normwerten der Thrombozyten $>50 \mathrm{Gpt} / \mathrm{l}$, $\mathrm{pH}>7$, Körpertempertemperatur $>35^{\circ} \mathrm{C}$, Serum-Calcium $>0,8 \mathrm{mmol} / 1$ wichtig. Tranexamsäure findet regelhaft Anwendung, da sie das Risiko einer Blutung verringert [92]. Rezente Studien weisen auf vermehrtes Auftreten von Krampfanfällen und thromboembolischen Ereignissen vor allem in höheren Dosierungen hin $[93,94]$.

Eine Fibrinogenkonzentration von unter 1,5-2 g/l ist mit einem erhöhten Blutungsrisiko vergesellschaftet und sollte nicht unterschritten werden. Fibrinogenkonzentrate sind hier die first-line Therapie [91]. Bei persistierender Blutungsneigung und erniedrigter Faktor XIII-Aktivität (beispielsweise <30\%) sollte dies mittels der Gabe von Faktor-XIII Konzentrat behoben werden [91]. Gefrorenes Frischplasma eignet sich für den gezielten Ersatz von Gerinnungsfaktoren nicht, da eine nicht ausreichend hohe Konzentration von Gerinnungsfaktoren gewährleistet werden kann. Prothrombinkomplexkonzentrate (PPSB) können bei verlängerter INR und Blutungsneigung gegeben werden, wobei die Empfehlungen nur unter Einnahme von Vitamin-K-Antagonisten eindeutig sind [91]. Rekombinanter Faktor VII (rFVIIa) wird nur in ausgewählten, mit anderen Mitteln nicht-beherrschbaren und lebensbedrohlichen Blutungssituationen als offlabel use eingesetzt.

Individuell gesteuerte impedanzaggregometrische bzw. thrombelastographische point-of-care Verfahren geben zusätzlich zu den sogenannten Standardgerinnungstests wie partielle Thromboplastinzeit (PTT) und der aktivierten partiellen Thromboplastinzeit (aPTT) Aufschluss über Fibrinogenpolymerisationsstörungen, Heparinwirkung, Hyperfibrinolysen, Faktorenmangelzustände und Thrombozytenfunktionsstörungen. Entscheidend ist die Sicherstellung einer ausreichenden hämostatischen Kapazität. Die definitive Versorgung von Blutungen, eventuell durch eine wiederholte chirurgische Intervention, kann aber auch notwendig sein.

Eine Homöostase der Körpertemperatur ist für eine funktionierende plasmatische Gerinnung sehr wichtig. Patienten, die nach einer offenen Versorgung einer traumatischen Aortenruptur mit oder ohne Einsatz der Herz-Lungen-Maschine postoperativ auf der Intensivstation behandelt werden, sind meist charakteristischerweise zunächst hypotherm. Die Regelmechanismen des homoiothermen Menschen sind gestört oder teilweise außer Kraft gesetzt. Der mensch- liche Körper benötigt einen Bereich um den Sollwert $37^{\circ} \mathrm{C}$, um Sauerstofftransport, Muskelaktivität und diverse Stoffwechselfunktionen gewährleisten $\mathrm{zu}$ können.

Patienten haben in der frühen postoperativen Phase nach Operationen an der Herz-Lungen-Maschine sehr häufig einen relativen oder absoluten Volumenmangel. Eine adäquate Volumentherapie zur Stabilisierung der hämodynamischen Situation stellt eine große Herausforderung für die weitere intensivmedizinische Behandlung dar. Die Wahl des geeigneten Volumenersatzmittels muss nach sorgfältiger Erwägung der Pathophysiologie des Volumenmangels erfolgen. Die mannigfaltigen Ursachen des Volumenmangels können unter anderem ein reaktives kapilläres Leck, ungenügender Ersatz von Blutverlusten, weiterbestehende Blutverluste, Drainageverluste, Temperaturschwankungen oder Nachwirkungen einer vorbestehenden präoperativen Diuretikabehandlung sein. Kristalloide Lösungen verteilen sich überwiegend im Extrazellulärraum, während kolloidale Lösungen eher intravasal verbleiben. Bei einem Volumenmangel ist der intravasale Mangel auszugleichen.

In Abwesenheit pulmonaler und anderer Kontraindikationen und nach Ausgleich des Temperaturhaushaltes sollte eine frühestmögliche Extubation oder Weaning angestrebt werden. Dies kann entscheidend für die Reduktion pulmonaler und anderer Komplikationen, einschließlich eines Delirs, sein [95].

Das Delirrisiko ist nach kardiochirurgischen Eingriffen mit einer Inzidenz von $26-52 \%$ besonders hoch [96]. Mit einem Delir ist auch eine erhöhte Letalität assoziiert. Ein richtig angewendetes Analgesie- und Sedierungsmanagement sowie tägliches Delirscreening mit validem Instrument sind enorm wichtig. Zudem scheinen Fast-track-Konzepte die Inzidenz für ein postoperatives Delir zu reduzieren [97]. Mehrfach wurde auch die sichere Durchführung frühzeitiger Mobilisierung und aktiver Physiotherapie beschrieben [98].

Acute Kidney Injuries (AKI) nach Versorgung von Aortenverletzungen sind häufig und multifaktoriell bedingt. Je nach Ausmaß der AKI ist sie mit einer erhöhten Mortalität vergesellschaftet [99]. Eine Dysbalance zwischen der Perfusion (VO2) der Nierenmedulla und dem Sauerstoffangebot (DO2) kann zu erheblichen Nierenschäden führen. Das renale DO2 kann intra- und postoperativ erheblich variieren und ein auch nur kurz andauerndes insuffizientes DO2 ist signifikant mit einem Auftreten eines AKI assoziiert. Studien haben gezeigt, dass hierbei der MAP-Wert weniger entscheidend ist als eine adäquate renale Perfusion [100]. Ein Sicherstellen eines adäquaten renalen DO2 und gutes hämodynamisches Management sind demzufolge wesentlich [101]. Rezente Kriterien der Kidney Disease Improving Global Outcomes (KDIGO) werden von den aktuellen Richtlinien empfohlen [102]. Diese betonen, dass ein Anstieg des Serumkreatinins bei bereits bestehendem AKI beginnt. Neuere 
Biomarker wie das Neutrophile Gelatinase-Assoziierte Lipocalin (NGAL) und das Interleukin-18 (IL-18) erscheinen vielversprechend bezüglich der früheren Erkennung, haben sich aber aus mehreren Gründen noch nicht klinisch etabliert. Rivers et al. legten im Rahmen ihrer Studie bei septischen Patienten einen Zielwert der Diurese $>0,5 \mathrm{ml} / \mathrm{kgKG} / \mathrm{h}$ fest [103]. Auf postoperative kardiochirurgische Patienten ist dieser Zielwert übertragbar. Der Diurese-Zielwert von $>0,5 \mathrm{ml} / \mathrm{kgKG} / \mathrm{h}$ ist nur ohne den Einsatz von Diuretika als adäquat zu betrachten. Der Beweis eines wirklichen Benefits von nephroprotektiven pharmakologischen Therapien ist bisher noch nicht erbracht. Es kann versucht werden, das Auftreten einer AKI durch präoperative Identifikation von Risikopatienten, Vermeidung modifizierbarer Risikofaktoren und eine frühzeitige Diagnosestellung zu verhindern.

Funding Open access funding provided by University of Innsbruck and Medical University of Innsbruck.

Interessenkonflikt T. Schachner, J. Oji-Zurmeyer, B. Rylski, S. Kapral, O. Stanger, G. Widmann, F. Schönhoff, M. Czerny und S. Jochberger geben an, dass kein Interessenkonflikt besteht.

Open Access Dieser Artikel wird unter der Creative Commons Namensnennung 4.0 International Lizenz (http:// creativecommons.org/licenses/by/4.0/deed.de) veröffentlicht, welche die Nutzung, Vervielfältigung, Bearbeitung, Verbreitung und Wiedergabe in jeglichem Medium und Format erlaubt, sofern Sie den/die ursprünglichen Autor(en) und die Quelle ordnungsgemäß nennen, einen Link zur Creative Commons Lizenz beifügen und angeben, ob Änderungen vorgenommen wurden.

\section{Literatur}

1. Carl M, Alms A, Braun J, et al. S3 guidelines for intensive care in cardiac surgery patients: hemodynamic monitoring and cardiocirculary system. Ger Med Sci.2010;8:Doc12.

2. Fabian TC, Richardson JD, Croce MA, Smith JS, Rodman G, Kearney PA, et al. Prospective study of blunt aortic injury: multicenter Trial of the American Association for the Surgery of Trauma. JTrauma. 1997;42:380-3.

3. Shackelford S, Nguyen L, Noguchi T, Sathyavagiswaran L, Inaba K, Demetriades D. Fatalities of the 2008 Los Angeles train crash: autopsy findings. Am J Disaster Med. 2011;6:127-31.

4. Pearson R, etal. Regional wall mechanics andblunt traumatic aortic rupture at the isthmus. Eur J Cardiothorac Surg. 2008;34:612-22.

5. Shkrum MJ, McClafferty KJ, Green RN, NowakES, Young JG. Mechanisms of aortic injury in fatalities occurring in motor vehicle collisions. J Forensic Sci. 1999;44:44-56.

6. Brundage SI, Harruff R, Jurkovich GJ, Maier RV. The epidemiology of thoracic aortic injuries in pedestrians. J Trauma. 1998:45:1010-4.

7. Arajärvi E, Santavirta S, Tolonen J. Aortic ruptures in seat belt wearers. JThorac Cardiovasc Surg. 1989;98:355-61.

8. Richens D, Kotidis K, Neale M, et al. Rupture of the aorta following road traffic accidents in the United Kingdom 1992-1999: the results of the co-operative crash injury study. Eur J Cardiothorac Surg. 2003;23:143-8.
9. Shkrum MJ, McClafferty KJ, Nowak ES, German A. Driver and front seat passenger fataliites associated with air bag deployment. Part 1: a Canadian study. J Forensic Sci. 2002;47:1028-34.

10. Sato Y, Ohshima T, Kondo T. Air bag injuries-a literature review in consideration of demands in forensic autopsies. Forensic Sci Int. 2002;128:162-7.

11. DeGuzman BJ, Morgan AS, Pharr WF. Aortic transection following airbag deployment. NEnglJ Med. 1997;337:573-4.

12. Wallis LA, Greaves I. Injuries associated with airbag deployment. Emerg Med J. 2002;19:490-3.

13. Parmley LF, Mattingly TW, Manion MC, et al. Nonpenetrating traumatic injury of the aorta. Circulation. 1958;17:1086-101.

14. Otte D, Facius T. Brand Sinjury mechanisms of aortic ruptures to vehicle occupants and vulnerable road users-an indepth-investigation over time. Forensic Biomech. 2017;8:1.

15. Fitzharris M, Franklyn M, Frampton R, Yang K, Morris A, Fildes B. Thoracic aortic injury in motor vehicle crashes: the effect of impact direction, side of body struck, and seat belt use. JTrauma. 2004;57:582-90.

16. Gotzen L, Flory PJ, Otte D. Biomechanics of aortic rupture at classical location in traffic accidents. Throac Cardiovasc Surg. 1980;28:64-8.

17. Neschis DG, Scalea TM, Flinn WR, Griffith BP. Blunt aortic injury. NEngl J Med. 2008;359:1708-16.

18. Richens D, Field M, Neale M, Oakley C. The mechanism of injury in blunt traumatic rupture of the aorta. Eur J Cardiothorac Surg. 2002;21:288-93.

19. Zhao R, Field ML, Richens D, Digges K. Blunt trauma and acute aortic syndrome: a three layer finite element model. Eur JCardiothorac Surg. 2008;34:623-9.

20. Siegel JH, Yang KH, Smith JA, Siddiqi SQ, Shah C, Maddali $\mathrm{M}$, et al. Computer simulation and validation of the Archimedes Lever hypothesis as a mechanism for aortic isthmus disruption in a case of lateral impact motor vehicle crash: a Crash Injury Research Engineering Network (CIREN) study. J Trauma. 2006;60:1072-82.

21. Crass JR, Cohen AM, Motta AO, Tomashefski JF, Weisen EJ. A proposed new mechanism of traumatic aortic rupture: the osseous pinch. Radiology. 1990;176:645-9.

22. LundwallJ.Themechanics of traumatic rupture of the aorta. Acta Pathol Microbiol Scand. 1964;62:34-6.

23. Field ML, RichensD. AnticipatoryValsalva-type response as a contributory factor in low impact blunt traumatic aortic rupture. Med Hypotheses. 2006;67:87-92.

24. Mohan D, Melvin JW. Failure properties of passive human aortic tissue. J Biomech. 1982;15:887-902.

25. Kroell C, Schneider D, Hahum A. Impact tolerance and response of the human thorax. 18th Stapp car crash conference. Pennsylvania: Society of Automotive Engineers; 1974.

26. Holzapfel GA. Determination of material models for arterial walls from uniaxial extension tests and histological structure. J Theor Biol. 2006;238:290-302.

27. Antonopoulos CN, Sfyroeras GS, Kallinis A, Kakisis JD, Liapis CD, Petridou ET. Epidemiology of concomitant injuries in traumatic thoracic aortic rupture: a meta-analysis. Vascular. 2014;22:395-405.

28. Mosquera VX, Marini M, Muñiz J, Asorey-Veiga V, Adrio-Nazar B, Boix R, et al. Traumatic aortic injury score (TRAINS): an easy and simple score for early detection of traumatic aortic injuries in major trauma patients with associated blunt chest trauma. Intensive Care Med.2012;38:1487-96.

29. Azizzadeh A, Keyhani K, Miller CC, Coogan SM, Safi HJ, Estrera AL. Blunt traumatic aortic injury: initial experience with endovascular repair. JVasc Surg. 2009;49:1403-8. 
30. Starnes BW, Lundgren RS, Gunn M, Quade S, Hatsukami TS, Tran NT, et al. A new classification scheme for treating blunt aortic injury. JVasc Surg. 2012;55:47-54.

31. Osgood MJ, Heck JM, Rellinger EJ, Doran SL, Garrard CL, Guzman RJ, et al. Natural history of grade I-II blunt traumatic aortic injury. JVasc Surg. 2014;59:334-41.

32. Rieger M, Sparr H, Esterhammer R, Fink C, Bale R, Czermak B, et al. Modern CT diagnosis of acute thoracic and abdominal trauma. Anaesthesist. 2002;51:835-42.

33. Mirvis SE, Bidwell JK, Buddemeyer EU, et al. Value of chest radiography in excluding traumatic aortic rupture. Radiology. 1987;163:487-93.

34. GutierrezA, Inaba K, Siboni S, EffronZ, Haltmeier T, JaffrayP, et al. The utility of chest X-ray as a screening tool for blunt thoracic aortic injury. Injury. 2016;47:32-6.

35. Smith MD, Cassidy JM, Souther S, Morris EJ, Sapin PM, Johnson SB, et al. Transesophageal echocardiography in the diagnosis of traumatic rupture of the aorta. N Engl J Med. 1995;332:356-62.

36. Vignon P, Gueret P, Vedrinne JM, Lagrange P, Cornu E, Abrieu $\mathrm{O}$, et al. Role of transesophageal echocardiography in the diagnosis and management of traumatic aortic disruption. Circulation. 1995;92:2959-68.

37. Hainer C, Böckler D, Bernhard M, Scheuren K, Stein KM, Rauch $\mathrm{H}$, et al. Blunt traumatic aortic injury: importance of transesophageal echocardiography. Anaesthesist. 2008;57:262-8.

38. MalhotraAK, FabianTC, Croce MA, WeimanDS, GavantML, Pate JW. Minimal aortic injury: a lesion associated with advancing diagnostic techniques. J Trauma. 2001;51:1042-8.

39. Lee WA, Matsumura JS, Mitchell RS, Farber MA, Greenberg RK, Azizzadeh A, etal.Endovascular repair of traumatic thoracic aortic injury: clinical practice guidelines of the Society for Vascular Surgery. J Vasc Surg. 2011;53:187-92.

40. Rabin J, Harris DG, Crews GA, Ho M, Taylor BS, Sarkar R, etal.Early aortic repair worsens concurrent traumatic brain injury. Ann ThoracSurg. 2014;98:46-51.

41. Mosquera VX, Marini M, Muñiz J, Gulias D, Asorey-Veiga V, Adrio-Nazar B, et al. Blunt traumatic aortic injuries of the ascending aorta and aortic arch: a clinicalmulticentre study. Injury. 2013;44:1191-7.

42. Schachner T. Acute aortic syndrome-when is operative treatment indicated? Dtsch Med Wochenschr. 2013;138:2375-8.

43. Murad MH, Rizvi AZ, Malgor R, Carey J, Alkatib AA, Erwin PJ, etal. Comparative effectiveness of the treatments for thoracic aortic transection. JVasc Surg. 2011;53:193-9.

44. Jonker FH, Verhagen HJ, Lin PH, Heijmen RH, Trimarchi S, Lee WA, et al. Open surgery versus endovascular repair of ruptured thoracic aortic aneurysms. J Vasc Surg. 2011;53:1210-6.

45. Trust MD, Teixeira PGR. Blunt Trauma of the Aorta, Current Guidelines. CardiolClin. 2017;35:441-51.

46. Fabian TC, Davis KA, Gavant ML, Croce MA, Melton SM, Patton JHJr, et al. Prospective study of blunt aortic injury: helical CT is diagnostic and antihypertensive therapy reduces rupture. Ann Surg. 1998;227:676-7.

47. Lam CR, Aram HH. Resection of the descending thoracic aorta for aneurysm; a report of the use of a homograft in a case and an experimental study. Ann Surg. 1951;134:743-52.

48. Pate JW, Cole FH, Walker WA, et al. Penetrating injuries of the aortic arch and its branches. Ann Thorac Surg. 1993;55:586-92.

49. Galli R, Pacini D, Di BR, et al. Surgical indications and timing of repair of traumatic ruptures of the thoracic aorta. Ann Thorac Surg. 1998;65:461-4.
50. Pate JW, Gavant ML, Weiman DS, et al. Traumatic rupture of the aortic isthmus: program of selective management. World J Surg. 1999;23:59-63.

51. Svensson LG, Antunes MD, Kinsley RH. Traumatic rupture of the thoracic aorta: a report of 14 cases and a review of the literature. SAfr Med J. 1985;67:853-7.

52. von Oppell UO, Dunne TT, De Groot MK, Zilla P. Traumatic aortic rupture: twenty-year metaanalysis of mortality and risk of paraplegia. Ann Thorac Surg. 1994;58:585-93.

53. Demetriades D, Velmahos GC, Scalea TM, Jurkovich GJ, Karmy-Jones R, Teixeira PG, et al. Operative repair or endovascular stent graft in blunt traumatic thoracic aortic injuries: results of an American Association for the Surgery of Trauma Multicenter Study. J Trauma. 2008;64:561-70.

54. Estrera AL, Gochnour DC, Azizzadeh A, Miller CC, Coogan S, Charlton-Ouw K, et al. Progress in the treatment of blunt thoracic aortic injury: 12-year single-institution experience. Ann Thorac Surg. 2010;90:64-71.

55. Bhaskar J, Foo J, Sharma AK. Clamp-and-sew technique for traumatic injuries of the aorta: 20-year experience. Asian Cardiovasc Thorac Ann. 2010;18:161-5.

56. Cardarelli MG, McLaughlin JS, Downing SW, Brown JM, AttarS, Griffith BP. Management of traumatic aortic rupture: a 30-year experience. Ann Surg. 2002;236:465-9.

57. Razzouk AJ, Gundry SR, Wang N, del Rio MJ, Varnell D, Bailey LL. Repair of traumatic aortic rupture: a 25 -year experience. Arch Surg. 2000;135:913-8.

58. Katz NM, Blackstone EH, Kirklin JW, et al. Incremental risk factors for spinal cord injury following operation for acute traumatic aortic transection. J Thorac Cardiovasc Surg. 1981;81:669-74.

59. VerdantA,PageA, CossetteR, etal.Surgery of the descending thoracic aorta: spinal cord protection with the Gott shunt. Ann ThoracSurg. 1988;46:147-54.

60. Hilgenberg AD, Logan DL, Akins CW, et al. Blunt injuries of the thoracic aorta. Ann Thorac Surg. 1992;53:233-8.

61. Pate JW, Fabian TC, Walker WA. Acute traumatic rupture of the aortic isthmus: repair with cardiopulmonary bypass. Ann ThoracSurg. 1995;59:90-8.

62. BouchartF, BessouJP, TableyA, Litzler PY, Haas-HubscherC, Redonnet M, et al. Acute traumatic rupture of the thoracic aorta and its branches. Results of surgical management. Ann Chir. 2001;126:201-11.

63. Verdant A. Contemporary results of standard open repair of acute traumatic rupture of the thoracic aorta. J Vasc Surg. 2010;51:294-8.

64 . Verdant A. Open versus endovascular repair for acute traumatic thoracic aortic rupture. J Thorac Cardiovasc Surg. 2012;143:1230.

65. Volodos NL, Karpovich IP, Shekhanin VE, Troian VI, Iakovenko LF. A case of distant transfemoral endoprosthesis of the thoracic artery using a self-fixing synthetic prosthesis in traumatic aneurysm. Grudn Khir. 1988;6:84-6.

66. Cindy M, Sabrina H, Kim D, Geert M, Inge F. Traumatic aortic rupture: 30 years of experience. Ann Vasc Surg. 2011;25:474-80.

67. Lebl DR, Dicker RA, Spain DA, Brundage SI. Dramatic shift in the primary management of traumatic thoracic aortic rupture. Arch Surg. 2006;141:177-80.

68. Karmy-Jones R, Ferrigno L, Teso D, Long WB, Shackford S. Endovascular repair compared with operative repair of traumatic rupture of the thoracic aorta: a nonsystematic review and a plea for trauma-specific reporting guidelines. JTrauma. 2011;71:1059-72.

69. Steuer J, Björck M, Sonesson B, Resch T, Dias N, Hultgren R, et al. Durability of endovascular repair in blunt traumatic 
thoracicaorticinjury: long-term outcomefromfour tertiary referral centers. Eur JVasc Endovasc Surg. 2015;50:460-5.

70. Zahn R, Erbel R, Nienaber CA, Neumann FJ, Nef H, Eggebrecht $\mathrm{H}$, et al. Endovascular aortic repair of thoracic aortic disease: early and 1-year results from a German multicenter registry. JEndovasc Ther. 2013;20:265-72.

71. Fanelli F, Dake MD. Standard of practice for the endovascular treatment of thoracic aortic aneurysms and type B dissections. Cardiovasc Intervent Radiol. 2009;32:849-60.

72. Matsumura JS, LeeWA, Mitchell RS, Farber MA, Murad MH, Lumsden AB, et al. Society for Vascular Surgery. The Society for Vascular Surgery Practice Guidelines: management of the left subclavian artery with thoracic endovascular aortic repair. JVasc Surg. 2009;50:1155-8.

73. Riesenman PJ, Farber MA, Mendes RR, Marston WA, Fulton JJ, Keagy BA. Coverage of the left subclavian artery during thoracic endovascular aortic repair. J Vasc Surg. 2007;45:90-4

74. Antonello M, Menegolo M, Maturi C, Dall'antonia A, Lepidi S, Frigo AC, et al. Intentional coverage of the left subclavian artery during endovascular repair of traumatic descending thoracic aortic transection. J Vasc Surg. 2013;57:684-90.

75. Lettinga-van de Poll T, Schurink GW, De Haan MW, et al. Endovascular treatment of traumaticrupture of thethoracic aorta. BrJ Surg. 2007;94:525-33.

76. Muhs BE, Balm R, White GH, et al. Anatomic factors associated with acute endograftcollapse after Gore TAG treatment of thoracic aortic dissection or traumatic rupture. J Vasc Surg. 2007;45:655-61.

77. Marcheix B, Dambrin C, BolducJP, etal. Endovascular repair of traumatic rupture of the aortic isthmus: midterm results. JThorac Cardiovasc Surg. 2006;132:1037-41.

78. Kato N, Dake MD, Miller DC, et al. Traumatic thoracic aortic aneurysm: treatment with endovascular stent-grafts. Radiology. 1997;205:657-62.

79. Tehrani HY, Peterson BG, Katariya K, et al. Endovascular repair of thoracic aortic tears. Ann Thorac Surg. 2006;82:873-7.

80. Svensson LG, Kouchoukos NT, Miller DC, et al. Expert consensus document on the treatment of descending thoracic aortic disease using endovascular stent-grafts. Ann Thorac Surg. 2008;85:S1-41.

81. Einstein AJ, Weiner SD, Bernheim A, Kulon M, Bokhari S, Johnson LL, et al. Multiple testing, cumulative radiation dose, and clinical indications in patients undergoing myocardial perfusion imaging. JAMA. 2010;304:2137-44.

82. Canaud L, Marty-Ané C, Ziza V, Branchereau P, Alric P. Minimum 10-year follow-up of endovascular repair for acute traumatic transection of the thoracic aorta. J Thorac Cardiovasc Surg. 2015;149:825-9.

83. Nation DA, Wang GJTEVAR. Endovascular repair of the thoracic aorta. Semin Intervent Radiol. 2015;32:265-71.

84. Schmittinger CA, Torgersen C, Luckner G, et al. Adverse cardiac events during catecholamine vasopressor therapy: a prospective observational study. Intensive Care Med. 2012;38:950-8.

85. DeBacker D, Creteur J, Dubois MJ, et al. Microvascular alterations in patients with acute severe heart failure and cardiogenic shock. Am Heart J. 2004;147:91-9.

86. Lima A, Jansen TC, van Bommel J, et al. The prognostic value of the subjective assessment of peripheral perfusion in criticallyill patients. Crit Care Med. 2009;37:934-8.

87. Wo CC, Shoemaker WC, Appel PL, et al. Unreliability of blood pressure and heart rate to evaluate cardiac output in emergency resuscitation and critical illness. Crit Care Med. 1993;21:218-23.

88. Kruse O, Gunnet N, Barford C. Blood lactate as a predictor for in-hospital mortality in patients admitted acutely to hospital: a systematic review. Scand J Trauma Resusc Emerg Med.2011;19:74.

89. Nichol AD, Egi M, Pettila V, et al. Relative hyperlactatemia and hospital mortality in critically ill patients: a retrospectivemulti-centre study. Crit Care. 2010;14:R25.

90. Murhy GJ, Pike K, Rogers CA, et al. Liberal or restrictive transfusion after cardiac surgery. N Engl J Med. 2015;372:997-1008.

91. Kozeck-Langenecker SA, Ahmed AB, Afshari A, et al. Management of severe perioperativebleeding: guidelines from theEuropean Society of Anaesthesiology: First update 2016. Eur JAnaesthesiol.2017;34:332-95.

92. CRASH-2 trial collaborators, ShakurH, Roberts I, Bautista R, Caballero J, Coats T, Dewan Y, et al. Effects of tranexamic acid on death, vascular occlusive events, and blood transfusion in trauma patients with significant haemorrhage (CRASH-2): a randomised, placebo-controlled trial. Lancet. 2010;376(9734):23-32

93. Hunt BJ. The current place of tranexamic acid in the management of bleeding. Anaesthesia. 2015;70:50-3.

94. Myles PS, Smith JA, Forbes A, et al. Tranexamic acid in patients undergoing coronary artery surgery. N Engl J Med. 2017;376:136-48.

95. Arenson BG, MacDonald LA, Grocott HP, et al. Effect of intensive care unit environment on in-hospital delirium after cardiac surgery. J Thorac Cardiovasc Surg. 2013;146:172-8.

96. Brown CH. Delirium in the cardiac surgical ICU. Curr Opin Anaesthesiol. 2014;27:117-22.

97. AWMF. S3-Leitlinie 001/012 Analgesie, Sedierung und Delirmanagement in der Intensivmedizin, aktueller Stand 08/2015.. https:/ /www.awmf.org/uploads/tx_szleitlinien/ 001-0121_S3_Analgesie_Sedierung_Delirmanagement Intensivmedizin_2015-08_01.pdf. Zugegriffen: 15. Jan. 2018.

98. Aneman A, Brechot N, Brodie D, et al. Advances in critical care management of patients undergoing cardiac surgery. Intensive Care Med. 2018;44:799-810.

99. Ambler GK, Coughlin PA, Hyes PD, et al. Incidence and outcomes of severe renal impairment following ruptured abdominal aortic aneurysm repair. Eur J Vasc Endovasc Surg. 2015;50:443-9.

100. Kandler K, Jensen ME, Nilsson J, et al. Arterial pressure during cardiopulmonary bypass is not associated with acute kidney injury. Acta Anaesthesiol Scand. 2015;59:625-31.

101. Li P, Qu LP, Qi D, et al. Significance of perioperative goaldirected hemodynamic approach in preventing postoperative complications in patients after cardiac surgery: a metaanalysis and systematic review. Ann Med. 2017;49:343-51.

102. Khwaja A. KDIGO clinical practice guidelines for acute kidney injury. Nephron Clin Pract. 2012;120:179-84.

103. Rivers E, Nguyen B, Havstad S, et al. Early goal-directed therapy in the treatment of severe sepsis and septic shock. NEngl J Med. 2001;345:1368-77.

104. Mangano DT, Tudor IC, Dietzel C. Multicenter Study of Perioperative Ischemia Research Group; Ischemia Research and Education Foundation. The risk associated with aprotinin in cardiac surgery. N Engl J Med. 2006;354:353-65.

Hinweis des Verlags Der Verlag bleibt in Hinblick auf geografische Zuordnungen und Gebietsbezeichnungen in veröffentlichten Karten und Institutsadressen neutral. 